EPJ Web of Conferences 37, 01028 (2012)

DOI: $10.1051 /$ epjconf/20123701028

(C) Owned by the authors, published by EDP Sciences, 2012

\title{
Semi-inclusive DIS Experiments Using Transversely Polarized Targets in Hall-A: Current Results and Future Plans
}

\author{
Kalyan Allada ${ }^{\mathrm{a}}$ on behalf of Hall-A E06-010 Collaboration \\ Thomas Jefferson National Accelerator Facility, Newport News, Virginia 23606, USA
}

\begin{abstract}
Measurement of single (SSA) and double spin asymmetries (DSA) in semiinclusive DIS reactions using polarized targets provide a powerful method to probe transverse momentum dependent parton distribution functions (TMDs). In particular, the experimentally measured SSA on nucleon targets can help in extracting the transversity and Sivers distribution functions of $u$ and d-quarks. Similarly, the measured DSA are sensitive to the quark spin-orbital correlations, and provide an access to the TMD parton distribution function $\left(g_{1 T}\right)$. A recent experiment conducted in Hall-A Jefferson Lab using transversely polarized ${ }^{3} \mathrm{He}$ provide first such measurements on "effective" neutron target. The measurement was performed using $5.9 \mathrm{GeV}$ beam from CEBAF and measured the target SSA/DSA in the SIDIS reaction ${ }^{3} \mathrm{He}^{\uparrow}\left(e, e^{\prime} \pi^{ \pm}\right) X$. The kinematical range, $x=0.19 \sim 0.34$, at $Q^{2}=1.77 \sim 2.73(\mathrm{GeV} / c)^{2}$, was focused on the valence quark region. The results from this measurement along with our plans for future high precision measurements in Hall-A are presented.
\end{abstract}

\section{Introduction}

In recent years, the study of transverse momentum dependent parton distribution functions (TMDs) has become one of most active areas of research in the hadronic physics community. TMDs provide new and fundamental information about the structure of the nucleon by imaging its partonic structure, dynamics, and spin-orbital couplings in three-momentum space. At leading twist there are eight TMD quark distributions [1]: three of them, the unpolarized $\left(f_{1}\right)$, the helicity $\left(g_{1}\right)$ and the transversity $\left(h_{1}\right)$ distributions are integrated over the transverse momenta of quarks, while the other five depend not only on the longitudinal momentum fraction $x$, but also on the parton transverse momentum $k_{T}$. While inclusive DIS experiments provide an access to the first two PDFs, semi-inclusive deep inelastic scattering (SIDIS) allows us to probe other six TMDs. Of these eight TMDs, transversity $\left(h_{1}\right)$, Sivers $\left(f_{1 T}^{\perp}\right)$, pretzelosity $\left(h_{1 T}^{\perp}\right)$, and $g_{1 T}$ can be accessed using a transversely polarized target, while $h_{1 L}^{\perp}$ can be accessed using a longitudinally polarized target.

The transversity distribution gives the probability of finding a transversely polarized parton inside a transversely polarized nucleon with certain longitudinal momentum fraction $x$ and certain transverse momentum $k_{T}$. The Sivers function [2] provides the number density of unpolarized partons inside a transversely polarized proton, and it requires wave function components with nonzero orbital angular momentum and thus provides information about the correlation between the quark orbital angular momentum (OAM) and nucleon spin. Furthermore, it is a (naive) T-odd function which relies on the final state interactions (FSI) experienced by the active quark in a SIDIS process. In contrast to $f_{1 T}^{\perp}$, the functions $g_{1 T}$ and $h_{1 L}^{\perp}$ are T-even, and thus do not require FSI to be nonzero. Nevertheless, they also require interference between wave function components that differ by one unit of OAM and thus require OAM to be nonzero. Finally, pretzelosity requires interference between wave function components that differ by two units of OAM (e.g. p-p or s-d interference). The combined wealth of information

\footnotetext{
a e-mail: kalyan@jlab.org
}

This is an Open Access article distributed under the terms of the Creative Commons Attribution License 2.0, which permits unrestricted use, distribution, and reproduction in any medium, provided the original work is properly cited. 
from all these functions could thus be invaluable for disentangling the spin orbit correlations in the nucleon wave function, providing important information about the quark orbital angular momentum, and for imaging of the nucleon in full momentum space.

Single spin asymmetries (SSA) in SIDIS reaction using a transversely polarized proton target were measure by the HERMES [4,5] and COMPASS [6] collaborations. Non-zero asymmetries were observed for both Collins and Sivers moments in the $\pi^{+}$channel. However, for the $\pi^{-}$channel the Collins asymmetry was non-zero, whereas Sivers asymmetry was small and consistent with zero within the statistical precision of these experiments.

The target single spin asymmetry from the SIDIS reaction, in the leading twist, is expressed as

$$
\begin{aligned}
A_{U T} & \equiv \frac{1}{\left|S_{T}\right|} \frac{Y^{\uparrow}\left(\phi_{h}, \phi_{S}\right)-Y^{\downarrow}\left(\phi_{h}, \phi_{S}\right)}{Y^{\uparrow}\left(\phi_{h}, \phi_{S}\right)+Y^{\downarrow}\left(\phi_{h}, \phi_{S}\right)} \\
& =A_{U T}^{\sin \left(\phi_{h}+\phi_{S}\right)} \sin \left(\phi_{h}+\phi_{S}\right)+A_{U T}^{\sin \left(\phi_{h}-\phi_{S}\right)} \sin \left(\phi_{h}-\phi_{S}\right)+A_{U T}^{\sin \left(3 \phi_{h}-\phi_{S}\right)} \sin \left(3 \phi_{h}-\phi_{S}\right)
\end{aligned}
$$

where $\phi_{S}$ and $\phi_{h}$ are the azimuthal angles of the produced hadron and the target spin as defined by the Trento convention [3], $S_{T}$ is the transverse polarization of the target with respect to the virtual photon direction, and $Y^{\uparrow \downarrow}\left(\phi_{h}, \phi_{S}\right)$ is the normalized yield for the up-down transverse spin direction of the target. The three terms correspond to the Collins, Sivers and pretzelosity asymmetries, respectively. Similarly, the beam-target double spin asymmetry, $A_{L T}$, gives an access to the $\cos \left(\phi_{h}-\phi_{S}\right)$ term. Recently, the JLab E060-010 experiment measured these asymmetry moments on a polarized ${ }^{3} \mathrm{He}$ target and extracted Collins and Sivers asymmetry moments using SSA [7], and $\mathrm{A}_{L T}$ moments using double spin asymmetries (DSA) [8]. A detailed description of this experiment is discussed below followed by our future plans for a precision measurement of these quantities.

\section{The E06-010 Experiment}

Experiment E06-010 [7,8] was conducted at Jefferson Lab in Hall A [10] from October 2008 until February 2009. The measurement was done by scattering off longitudinally polarized electrons on a transversely polarized ${ }^{3} \mathrm{He}$ target [10] in the SIDIS reaction ${ }^{3} \mathrm{He}\left(e, e^{\prime} h\right) X$, where $h$ is a charged hadron either pion or kaon. An electron beam with energy of $5.9 \mathrm{GeV}$ and an average current of $12 \mu \mathrm{A}$ was used. The beam helicity was flipped at $30 \mathrm{~Hz}$. The beam polarization was periodically measured using a Moller polarimeter. The the average polarization was $(76.8 \pm 3.5) \%$. For the SSA, unpolarized beam was achieved by summing together the two electron helicity states. The scattered electrons were detected in the BigBite Spectrometer at an angle of $30^{\circ}$ to the beam-right side. The momentum range for this spectrometer was 0.6-2.5 GeV/c. The BigBite detector package consisted of a dipole magnet, three multi-wire drift chambers (MWDC) for tracking, a scintillator plane for timing and a lead glass calorimeter for particle identification (PID). The solid angle acceptance was $64 \mathrm{msr}$. The coincident charged hadrons were detected in the high resolution spectrometer (HRS) [10] with a central momentum of $2.35 \mathrm{GeV} / \mathrm{c}$ and at an angle of $16^{\circ}$ to the beam-left. The HRS has a momentum acceptance of $\pm 4.5 \%$ and a angular acceptance of $6 \mathrm{msr}$. The spectrometer detector package contained drift chambers for tracking, two scintillator planes for trigger and time-of-flight, and a gas Čerenkov counter and a two layer electromagnetic calorimeter for PID. Additionally, a ring imagining Čerenkov (RICH) and an aerogel Čerenkov were used for hadron identification.

A polarized ${ }^{3} \mathrm{He}$ target was used as an effective neutron target. In the ground state ${ }^{3} \mathrm{He}$ wave function is dominated by the $S$ state, where the two proton spins cancel and the ${ }^{3} \mathrm{He}$ polarization is effectively carried by the single neutron. The ${ }^{3} \mathrm{He}$ nuclei was polarized with $\mathrm{Rb}-\mathrm{K}$ mixture in the cell using a spin-exchange optical pumping method [11]. Three sets of orthogonal Helmholtz coils were used to orient the target polarization direction in either horizontal or vertical direction in the plane transverse to the beam momentum. The target was made of $40 \mathrm{~cm}$ long glass tube filled with ${ }^{3} \mathrm{He}$ gas at $10 \mathrm{~atm}$ pressure. This allowed us to reach the polarized neutron luminosity of $10^{36} \mathrm{~cm}^{-2} \mathrm{~s}^{-1}$. The target spin was automatically flipped every 20 minutes using adiabatic fast passage [12] method to reduce the systematic uncertainties and increase the azimuthal angular coverage. The Nuclear Magnetic Resonance(NMR) was used to measure the polarization with each spin flip, which was calibrated 


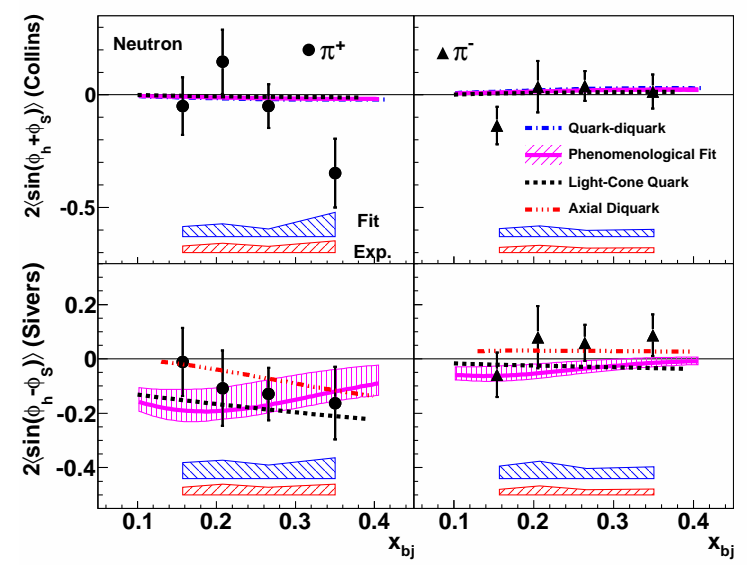

Fig. 1. The extracted neutron Collins (top) and Sivers (bottom) moments vs $x_{b j}$ for the electroproduction of $\pi^{+}$ (left) and $\pi^{-}$(right) from ${ }^{3} \mathrm{He}$ target. The bands below the data points represent the experimental and model uncertainties, which are labeled as Exp and Fit, respectively.

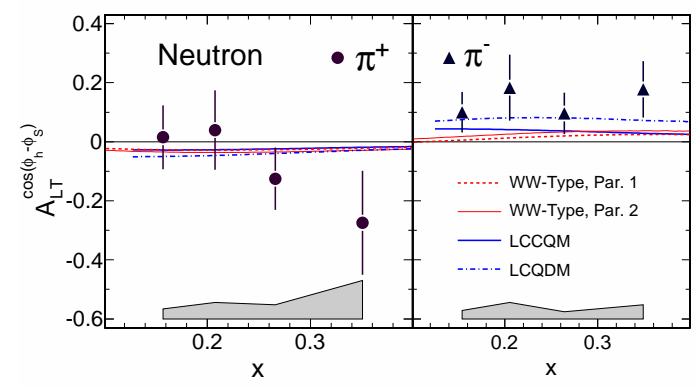

Fig. 2. The neutron $A_{L T}$ asymmetry for $\pi^{+}$(left) and $\pi^{-}$(right) vs $x_{b j}$ extracted from ${ }^{3} \mathrm{He}$ asymmetries and compared with model calculations.

using electron paramagnetic resonance [13] and a known polarization signal from an identical water cell. The average in-beam target polarization was $(55.4 \pm 2.8) \%$.

\section{Results}

The SIDIS event selection was done using PID and kinematic cuts. The following kinematical cuts were used: $Q^{2}>1.0(\mathrm{GeV} / \mathrm{c})^{2}, W>2.3 \mathrm{GeV} / \mathrm{c}^{2}$ and missing mass $W^{\prime}>1.6 \mathrm{GeV} / \mathrm{c}^{2}$. The Collins, Sivers and $\mathrm{A}_{L T}$ moments were extracted using two independent methods: (i) a local-pair binningfitting [7], where the moments are obtained by fitting asymmetries in the $2 \mathrm{D}\left(\phi_{h}, \phi_{S}\right)$ bins, (ii) an unbinned maximum likelihood method [8]. A small amount of nitrogen is used inside the target cells to reduce depolarization effects. The obtained moments were corrected for the $\mathrm{N}_{2}$ dilution in the target which was about $10 \%$. The neutron Sivers and Collins moments, and $\mathrm{A}_{L T}$ asymmetry were extracted from the ${ }^{3} \mathrm{He}$ results using the effective polarization method [15] as discussed in [7]. The method takes into account the initial-state nuclear effects. The proton dilution in the ${ }^{3} \mathrm{He}$ target was obtained by taking the ratio of yields for unpolarized hydrogen and ${ }^{3} \mathrm{He}$ targets in the SIDIS kinematics.

The results for the extracted neutron Collins and Sivers moments from the ${ }^{3} \mathrm{He}$ asymmetries are shown in Fig. 1 in the top and bottom panels, respectively. The left-hand side of the plot shows the results for the $\pi^{+}$and the right-hand side shows the results for $\pi^{-}$. The error bars on the points indicate 


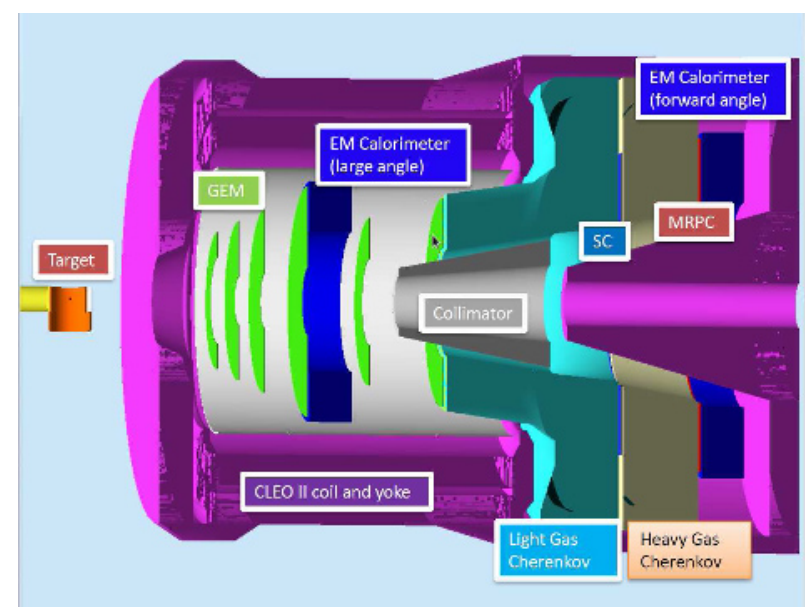

Fig. 3. A conceptual layout of the SoLID spectrometer and the polarized target in the proposed experiment. The sub-detectors are labeled with colors corresponding to the detector regions.

the statistical precision of the data, whereas the bands on the bottom show the experimental (Exp) and Fitting (Fit) systematic uncertainties. The total experimental uncertainties are less than $25 \%$ of the statistical uncertainty in each bin. The fitting uncertainties result from ignoring the pretzelosity and higher twist terms in the extraction of the moments. The data are compared with a phenomenological fit [14] and model calculations, which include a light-cone constituent quark model (LCCQM) [16,17] and quark-diquark calculations $[18,19]$. Both the data and the calculations indicate that the Collins asymmetries are small, though the data at $x_{b j}=0.34$ is more negative at the $2 \sigma$ level. For the Sivers moments, the $\pi^{+}$asymmetries favor negative values, whereas the $\pi^{-}$results are consistent with zero within the uncertainties.

The extracted $A_{L T}$ results of neutron from the ${ }^{3} \mathrm{He}$ asymmetries are shown in Fig. 2 along with model calculations, which include LT Wandzura-Wilczek (WW)-type approximations using parameterizations from Refs. [20,23], a LCCQM [16,17] and a light-cone quark-diquark model (LCQDM) [21]. The extracted asymmetries are consistent with zero for positive pions. The negative pion asymmetries are positive at the level of $2.8 \sigma$ with all four bins averaged together. This observation is consistent with the model predictions in sign but favors a larger magnitude.

\section{Future Experiments in Hall-A}

The main limitation of the E06-010 experiment was incomplete azimuthal angular acceptance which led to increase in the overall systematic error when extracting various azimuthal asymmetry moments. In order to overcome this limitation and to perform a very precise mapping of SSA and DSA in 4dimensions $\left(x, Q^{2}, P_{T}, z\right)$, three new experiments have been proposed in Hall-A using a new device: two measurements using polarized ${ }^{3} \mathrm{He}$ (transverse and longitudinal) target [24,25] and one with a transversely polarized $\mathrm{NH}_{3}$ (proton) target [26]. These measurement on proton and "effective" neutron targets will provide the most precise SIDIS data, and help in extracting the following TMDs: $h_{1}$,

$f_{1 T}^{\perp}, h_{1 T}^{\perp}, g_{1 T}, h_{1 L}^{\perp}$. The proposed experimental setup consists of the Solenoidal Large Intensity Device (SoLID) and a polarized target as shown in Fig. 3. The SoLID spectrometer has a full $2 \pi$ azimuthal angular coverage, which is essential to control the systematic uncertainties in extracting various azimuthal moments from the measured asymmetries. The polarized nucleon luminosity for the ${ }^{3} \mathrm{He}$ target is about $10^{36}$ nucleons $/ \mathrm{cm}^{2} / \mathrm{s}$ while that for $\mathrm{NH}_{3}$ target it is $10^{35}$ nucleons $/ \mathrm{cm}^{2} / \mathrm{s}$. Two different beam energies, $11 \mathrm{GeV}$ and $8.8 \mathrm{GeV}$, will be used to measure the asymmetries. The lower beam energy will provide precision data on the radiative corrections along with increased $\mathrm{Q}^{2}$ coverage. The SoLID detector consists of two regions. The forward region with polar angle coverage of $8.5^{\circ}$ to $16^{\circ}$ has a total solid angle of $95 \mathrm{msr}$, and covers a momentum range from $1 \mathrm{GeV} / \mathrm{c}$ to $7 \mathrm{GeV} / \mathrm{c}$ for both electrons and 


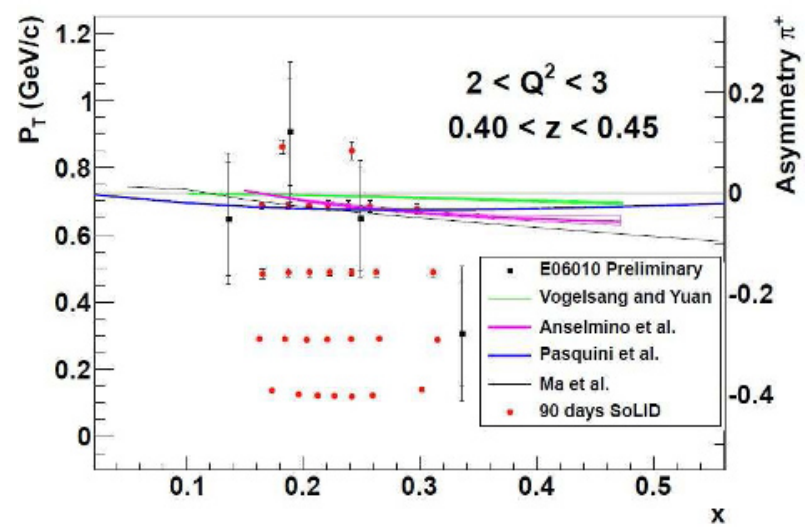

Fig. 4. Projected results for $\pi^{+}$Collins asymmetry in one bin of $Q^{2}$ and $z$. The y-axis on the left side shows $P_{T}$ coverage and $\mathrm{y}$-axis shows on the right side shows the scale of the asymmetry. The data from the Hall-A $6 \mathrm{GeV}$ experiment (E06-010) is shown in black point with large errors.

pions. Tracking will be done by a set of GEM detectors and particle identification will be provided by the combination of a gas Čerenkov, an aerogel counter, and an electromagnetic calorimeter. A thin layer of scintillator and one layer of Multi-gap Resistive Plate Chamber (MRPC) will be used for the timing information. The large angle region with polar angle coverage of $16^{\circ}$ to $25^{\circ}$ has a solid angle of $280 \mathrm{msr}$, and is mainly used for the electron detection in the momentum range of 4 to $6 \mathrm{GeV} / \mathrm{c}$. GEMs and an electromagnetic calorimeter will be used in this region to provide accurate momentum and energy reconstruction of the scattered electron.

Figure 4 shows the projected results for the $\pi^{+}$Collins asymmetry in one bin of $Q^{2}$ and $z$ that can be obtained in 90 days with a ${ }^{3} \mathrm{He}$ target. The theoretical predictions of Collins asymmetries are from Anselmino et al. [27], Vogelsang and Yuan [28] and Pasquini [29]. The X-axis shows the bjorken- $x$, y-axis on the left side shows the $P_{T}$ coverage, and y-axis on the right side shows the scale of the asymmetries. The overall kinematic coverage is as follows: $P_{T}$ from $0-1.6 \mathrm{GeV} / \mathrm{c}, Q^{2}$ from 1-8 $(\mathrm{GeV} / \mathrm{c})^{2}, z$ from $0.3-0.7$, and $x$ from $0.05-0.65 . P_{T}$ and $z$ coverage is divided into 8 bins each, and the $Q^{2}$ range is divided into 6 bins. The combined data from two beam energies will be divided into a total of 1400 bins for the SIDIS measurement with a ${ }^{3} \mathrm{He}$ target. This will allow us to study the measured asymmetries in 4 dimension $\left(x, Q^{2}, z, P_{T}\right)$. Along with the SSA, the DSA data will allow us to measure $A_{L T}$, which is related to the $g_{1 T}$ distribution. Moreover, SIDIS with longitudinally polarized beam on a longitudinally polarized target will provide $A_{L L}$ and $A_{U L}$ data which will be used to extract $g_{1 L}$ and $h_{1 L}^{\perp}$, respectively.

\section{Summary}

We have presented the first results of SSA and DSA measurements of charged pions in SIDIS reaction using a transversely polarized ${ }^{3} \mathrm{He}$ target. The extracted neutron Collins moments from the ${ }^{3} \mathrm{He}$ asymmetries were small and consistent with model calculations. The extracted Sivers moments favors negative values for $\pi^{+}$, whereas for $\pi^{-}$it is close to zero. For the first time a non-zero signal is observed for the $g_{1 T}$ moments in $\pi^{-}$channel. Future SIDIS measurements at Jefferson Lab will provide the most precise 4-D $\left(x, Q^{2}, z, P_{T}\right)$ data of SSA and DSA on both proton and "effective" neutron targets. The combined analysis of the data from these measurements will help in extracting various TMDs for both $\mathrm{u}$ and d-quarks. This will also enable us to do a model independent extraction of the $\mathrm{u}$ and d-quark tensor charges within a $10 \%$ accuracy. Measurement of Sivers, pretzelosity and $g_{1 T}$ distribution functions will enable a comprehensive understanding of quark orbital angular momentum, its relativistic effects and spin-orbit correlations. 


\section{Acknowledgments}

This work is supported by U.S. Department of Energy. Jefferson Science Associates, LLC operates the

Thomas Jefferson National Accelerator Facility under the contract DE-AC05-06OR23177.

\section{References}

1. A. Bacchetta. et al. JHEP (2007),02 093.

2. D. Sivers. Phys. Rev. D41 (1990) 83.

3. A. Bacchetta. Phys. Rev. D70 (2004) 117504.

4. A. Airapetian. et al. Phys. Rev. Lett. 94 (2005) 012002.

5. A. Airapetian. et al. Phys. Lett. B 693 (2010) 11.

6. M. Alekseev et al. Phys. Lett. B 692 (2010) 240.

7. X. Qian. et al. Phys. Rev. Lett. 107 (2011) 072003.

8. J. Huang. et al. Phys. Rev. Lett. 108 (2012) 052001.

9. A. M. Kotzinian and P. J. Mulders. Phys. Rev. D54 (1996) 1229.

10. J. Alcorn et al. Nucl. Instrum. Meth. A522 (2004) 294.

11. E. Babcock et al. Phys. Rev. Lett. 91 (2003) 123003.

12. A. Abragam. Principles of Nuclear Magnetism, Oxford University, New York, 1961.

13. M. V. Romalis and G. D. Cates. Phys. Rev. A58 (1998) 3004.

14. M. Anselmino et al. Phys. Rev. D75 (2007) 054032.

15. S. Scopetta et al.. Phys. Rev. D75 (2007) 054005.

16. S. Boffi et al. Phys. Rev. D79 (2009) 094012.

17. B. Pasquini, S. Cazzaniga, and S. Boffi. Phys. Rev. D78 (2008) 034025.

18. J. She and B. Q. Ma. Phys. Rev. D83 (2011) 037502.

19. B. Q. Ma, I. Schmidt, and J. J. Yang. Phys. Rev. D65 (2002) 034010.

20. A. Kotzinian, B. Parsamyan, and A. Prokudin. Phys. Rev. D73 (2006) 114017.

21. J. Zhu and B. Q. Ma. Phys. Lett. B696 (2011) 246.

22. H. Gao et al. Eur. Phys. J. Plus 126 (2011) 2.

23. A. Prokudin, private communication.

24. JLab proposal PR-12-10-006

http: //www. jlab.org/exp_prog/proposals/10/PR12-10-006.pdf.

25. JLab proposal PR-12-11-007

http://www.jlab.org/exp_prog/PACpage/PAC37/proposals/Proposals/NewProposals/PR-11-007.pdf 26. JLab proposal PR-12-11-108

http: //www.jlab.org/exp_prog/proposals/11/PR12-11-108.pdf.

27. M. Anselmino and A. Prokudin, private communication.

28. W. Vogelsang and F. Yuan, private communication

29. B. Pasquini, private communication 